\section{Jean-Claude Martinou}

\section{ADRESSE}

I.-C. Martinou: directeur de recherche. Glaxo, Institut de biologie moléculaire, 14 , chemin des Aulx, 1228 Plan-Les-Ouates, 1228 Genève, Suisse.

$\mathrm{m} / \mathrm{s} n^{\circ} 3$, vol. $11, \operatorname{mars} 95$

\title{
La mort cellulaire programmée dans le système nerveux
}

La mort cellulaire programmée ou apoptose, processus au cours duquel la cellule participe activement à sa propre destruction, est une étape essentielle du développement des organismes multicellulaires, très conservée au cours de l'évolution. Dans le système nerveux, la moitié des neurones meurent au cours de la synaptogenèse, en partie par défaut de facteurs trophiques qui supprimeraient un signal intrinsèque déclenchant le suicide. La protéine $\mathrm{Bcl}-2$ s'oppose à l'apoptose, sans doute par sa fonction antioxydante et par son inhibition de l'activité des protéases à cystéine ICE (interleukin $1 \beta$ comverting enzyme) et Nedd-2/ICH-1; les cibles et la régulation de ces protéases qui favorisent l'apoptose sont encore inconnues. Un dérèglement de l'équilibre entre ces protéines semble impliqué dans de nombreuses maladies humaines, en particulier dans des maladies neurodégénératives.

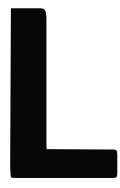

a mort cellulaire programmée, ou apoptose, est un processus au cours duquel la cellule participe activement à sa propre destruction. Cette autodestruction s'accompagne, presque toujours, de changements morphologiques caractéristiques, décrits il y a près de vingt ans par Wyllie et Kerr [1] (figure 1). Elle joue un rôle fondamental dans le développement de la plupart des organismes pluricellulaires et se trouve impliquée dans de nombreuses maladies humaines comme le cancer, le SIDA ou encore les maladies dégénératives. Dans cette revue, l'accent sera mis sur son rôle dans le développement du système nerveux, sur l'identification des intervenants dans ce programme et finalement sur son implication éventuelle dans les maladies neurodégénératives.
Mort de neurones au cours du développement chez le ver

Les travaux réalisés chez le nématode C. elegans plaident en faveur de l'existence d'un programme génétique de mort cellulaire. Chez cet invertébré, 131 des 1090 neurones produits durant la neurogenèse meurent après la phase de prolifération [2]. Ce processus d'élimination implique au moins 11 gènes et peut être divisé en trois étapes: mort du neurone, phagocytose et dégradation des cadavres cellulaires. Deux gènes, ced-3 et ced-4, sont impliqués dans la phase de mort cellulaire [3, 4]. Une mutation entraînant une perte d'activité de l'un des deux gènes bloque la mort, ce qui démontre que la fonction des deux gènes est requise pour l'accom- 


\section{RÉFÉRENCES}

1. Wyllie AH, Kerr JFR, Currie AR. Cell death: the significance of apoptosis. Int Rev Cytol 1980; 68: 251-306.

2. Ellis RE, Yuan J, Horvitz HR. Mechanisms and functions of cell death. Annu Rev Cell Biol 1991; 7: 663-98.

3. Yuan J, Horvitz HR. The Caenorhabditis ele gans cell death gene ced 4 encodes a novel protein and is expressed during the period of extensive programmed cell death. Deve lopment 1992;116: 309-20.

4. Labouesse M. C. elegans, les promesses d'un petit animal intelligent: "small is beautiful ". médecine/sciences 1994; 10:337 41 .

5. Hentgartner MO, Ellis RE, Horvitz HR. C. elegans gene ced-9 protects cells from programmed cell death. Nature 1992; $356: 494$ 8.

6. Denis $\mathrm{H}$, Mignotte B. L'apoptose dérive t-elle de la mort nucléaire programmée mise en cuvre par les protistes? médecine/sciences $1994 ; 10$ : 687-95.

7. Oppenheim RW. Cell death during development of the nervous system. Annu Rev Neurosci 1991 ; 14: 453-501.

8. Catsicas S, Thanos S, Clarke PGH. Major role for neuronal death during brain development: refinement of topographical connections. Proc Natl Acad Sci USA 1987; $84: 8165-8$.

9. Raff MC. Social controls on cell survival and death: an extreme view. Nature 1992 $356: 397-400$

10. Clarke PGH. Developmental cell death morphological diversity and multiple me chanisms. Anat Embryol 1990; 181: 195-213.

11. Hengartner MO, Horvitz HR. C. elegans cell survival gene ced-9 encodes a functional homolog of the mammalian proto-oncogene Bcl-2. Cell 1994; 76 : 665-76.

12. Tsujimoto $\mathrm{Y}$, Finger LR, Tunis J Nowell PC, Croce CM. Cloning of the chro mosomic breakpoint of neoplastic B cells with the $t(14 ; 18)$ chromosome translocation. Science $1984 ; 226$ : $1097-9$

13. Garcia I, Martinou I, Tsujimoto Y, Martinou JC. Prevention of programmed cell death of sympathetic neurons by the Bcl-2 plissement de la mort cellulaire programmée. Un autre gène, ced-9, s'oppose aux effets des gènes ced-3 et ced$4[4,5]$. Des mutants avec une surexpression de ce gène présentent le même phénotype que les mutants ced-3 et ced-4. Par ailleurs, une perte d'activité de ce gène entraîne la mort de neurones qui, normalement, ne dégénèrent pas, suggérant que les neurones survivent grâce à l'inhibition permanente, par Ced-9, d'un programme de mort cellulaire intrinsèque.

Ces études se sont révélées d'une importance capitale pour la compréhension de la mort cellulaire programmée chez les vertébrés. En effet, il est apparu que certains des mécanismes fondamentaux de la mort cellulaire programmée sont conservés au cours de l'évolution [6]. En témoignent l'homologie de structure et la conservation de la fonction qui existent entre les produits des gènes du nématode et ceux des vertébrés que nous décrirons plus loin. Toutefois, la mort cellulaire programmée des vertébrés implique d'autres mécanismes que ceux décrits chez le nématode. Avec l'évolution, le niveau de complexité du processus s'est accru, tant par la duplication des gènes participant à la mort cellulaire programmée que par la diversité des mécanismes de contrôle du processus. Par exemple, la part des facteurs épigénétiques dans la mort cellulaire programmée est plus grande chez les vertébrés que chez $C$. elegans.

\section{La mort cellulaire programmée dans le système nerveux des vertébrés}

La mort cellulaire programmée a été amplement étudiée dans le système nerveux des vertébrés depuis les travaux de Rita Levi-Montalcini et Victor Hamburger. Elle intervient durant une période bien précise du développement appelée période de mort cellulaire spontanée ou physiologique [7] et servirait à éliminer les neurones qui auraient fait des erreurs de connexion synaptique [8]. Chez les vertébrés, $50 \%$, en moyenne, des neurones dégénèrent au cours de la période périnatale. L'ampleur du phénomène varie selon les différentes populations de neurones.
Il est contrôlé en majeure partie par des facteurs trophiques rétrogrades spécifiques sécrétés au niveau des territoires d'innervation. La survie des neurones dépendrait de leur capacité d'accéder à une quantité suffisante de facteurs neurotrophiques: ceux qui reçoivent suffisamment de facteurs neurotrophiques survivent, les autres dégénèrent [9]. Des études morphologiques de neurones en voie de dégénérescence ont permis d'établir l'existence, à côté de la mort de type apoptotique classique, d'une grande diversité morphologique de morts cellulaires [10]. Cette diversité suggère l'existence d'une multiplicité de mécanismes moléculaires de l'apoptose dans le système nerveux. Toute mort de neurone ultérieure à la période de mort cellulaire physiologique peut être considérée comme pathologique ou liée au vieillissement. Elle est un des signes pathognomoniques des maladies neurodégénératives comme la maladie d'Alzheimer ou la maladie de Parkinson. La nature de la mort cellulaire qui intervient dans ces maladies sera discutée plus loin.

\section{Bcl-2, prototype d'une famille de protéines antiapoptotiques}

Chez les vertébrés, le gène homologue du gène ced-9 du ver est nommé $b c l-2[4,11]$. Le gène $b c l-2$ a été identifié au point de cassure chromosomique de la translocation entre les chromosomes 14 et 18 , une translocation que l'on rencontre dans la majorité des lymphomes folliculaires de type B $\left(m / s n^{\circ} 1, v o l .7, p .88\right)$ [12]. La surexpression de Bcl-2 dans les lymphocytes B qui résulte de cette translocation est responsable d'un allongement anormal de la durée de vie de ces cellules. Cela a donné naissance à l'idée que la croissance de certaines tumeurs serait due, non pas à une prolifération anormale de cellules, mais plutôt à l'accumulation de cellules dont le programme de mort cellulaire est défectueux.

Bcl-2 est capable d'augmenter la survie de nombreux types cellulaires. Nous avons montré que des neurones sympathiques dont la survie dépend du NGF pouvaient, grâce à Bcl-2, survivre environ sept jours en 


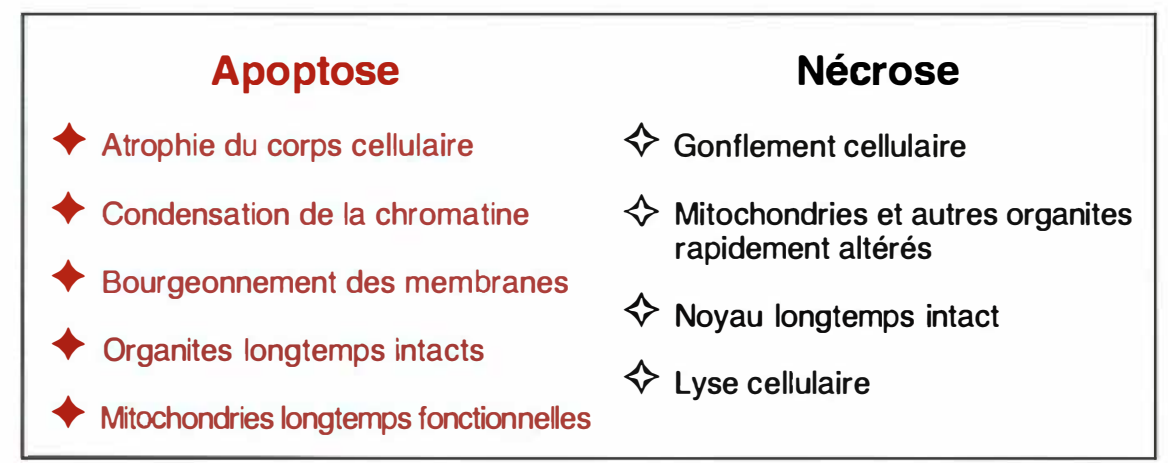

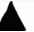

Figure 1. Critères morphologiques permettant de distinguer la mort de type apoptotique de la mort de type accidentel ou nécrotique.

Figure 2. Antagonisme Bax-Bcl-2: “le yin et le yang ». Les protéines Bcl2 et Bax peuvent former, soit des homodimères (Bcl-2/Bcl-2, Bax/Bax), soit des hétérodimères (Bax/Bcl-2). La survie cellulaire est associée à la présence des dimères Bax/Bcl-2. Comment agissent ces protéines? Dans cette figure, nous présentons deux modèles pour la fonction de Bax et de Bcl-2. Modèle A: Bcl-2 bloque un programme de mort cellulaire. Bax inhibe la fonction de Bcl-2. Modèle B: Bax active un programme de mort cellulaire. Bcl-2 inhibe l'action de Bax.

culture en absence de NGF $(\mathrm{m} / \mathrm{s}$ $n^{\circ} 10$, vol. 8, p. 1113) [13]. De nombreuses protéines présentant une analogie de structure avec Bcl-2 ont été récemment identifiées. Certaines protéines ont des fonctions antiapoptotiques - c'est le cas de Bcl-xl [14] d'autres, au contraire, s'opposent à l'effet protecteur de Bcl-2, c'est le cas de $\operatorname{Bax}\left(m / s n^{\circ} 11\right.$, vol. 9, p. 1268) [15]. Une analogie de structure réside dans deux domaines d'une dizaine d'acides aminés situés dans la région C-terminale. En outre, la plupart de ces protéines possèdent, dans la région C-terminale, une séquence d'acides aminés hydrophobes qui leur permet de s'ancrer dans différentes membranes (nucléaire, mitochondriale, réticulum endoplasmique) [16]. Le gène $b c l-x$ code pour deux protéines, $\mathrm{Bcl}-\mathrm{xl}$ et $\mathrm{Bcl}-\mathrm{xs}$, résultant d'un épissage alternatif des séquences d'homologie. Bcl-xl, la forme longue de la protéine, possède, comme Bcl-2, des propriétés antiapoptotiques [14]. Elle protège les lymphocytes et les neurones de la mort cellulaire induite par la privation en facteurs neurotrophiques. Bcl-xs, la forme courte, est capable de s'opposer à la fonction de $\mathrm{Bcl}-2$ par un mécanisme qui n'a pas encore été élucidé [17].

Les gènes $b c l-2$ et $b c l-x$ sont exprimés dans la plupart des neurones à des niveaux et à des temps différents. L'expression de $b c l-2$ est importante au cours de l'embryogenèse, durant la phase de prolifération et lors de la phase de mort cellulaire, puis elle chute dans les neurones adultes. L'expression de $b c l-x$ est importante non seulement dans le cerveau embryonnaire, mais aussi dans le cerveau adulte. La fonction des protéines Bcl2 et $\mathrm{Bcl}-\mathrm{x}$ durant le développement du système nerveux est encore inconnue. L'inactivation du gène $b c l-2$ chez la souris n'entraîne aucune anomalie notable du développement du système nerveux [18]. Ce résultat peut s'expliquer par un effet supplétif de Bcl-xl et peut-être même d'autres protéines antiapoptotiques encore inconnues. Dans le but d'étudier le rôle de Bcl-2 lors du développement, nous avons produit des souris transgéniques dont tous les neurones surexpriment $b c l-2$ dès le début de la période de mort cellulaire [19]. Nous avons remarqué que différentes régions du système nerveux de ces souris transgéniques renfermaient $40 \%$ à $50 \%$ de neurones de plus que la normale. Toutefois, au sein d'une population donnée de neurones, nous avons noté qu'une proportion de neurones dégénéraient normalement, malgré l'abondance de Bcl-2.

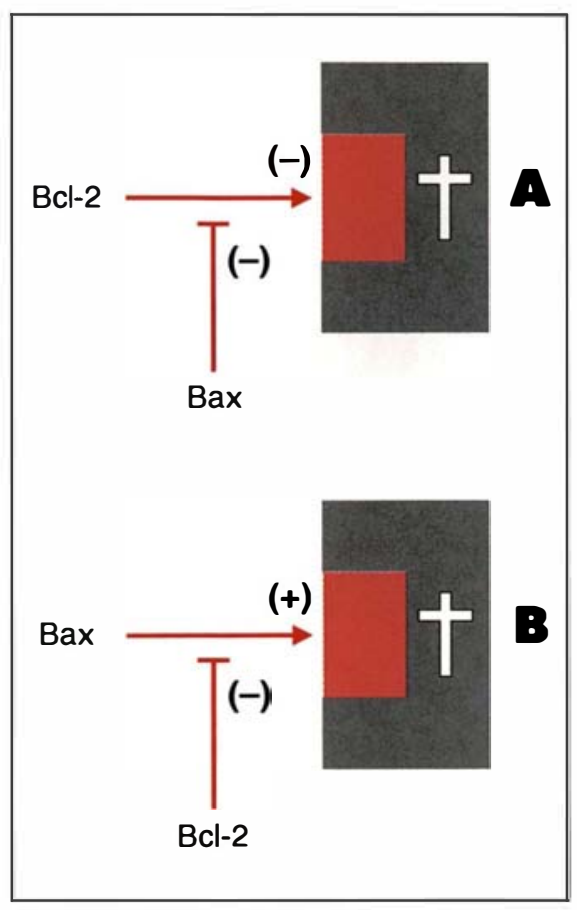

Ces résultats suggèrent qu'existent différents mécanismes de mort cellulaire, certains dépendants de Bcl-2, d'autres indépendants.

En conclusion, nous pensons que $\mathrm{Bcl}-2$ et $\mathrm{Bcl}-\mathrm{x}$ jouent un rôle important dans la sélection de certains neurones lors de la phase de mort cellulaire physiologique. Les neurones qui exprimeraient $\mathrm{Bcl}-2$ et $\mathrm{Bcl}-\mathrm{x}$ feraient l'objet d'une sélection positive, les autres seraient éliminés [20]. Ce type de sélection s'apparenterait à la sélection à laquelle sont soumis les lymphocytes dans les centres germinatifs des follicules secondaires et pour laquelle le rôle de Bcl-2 est bien établi. Pour l'instant, aucune étude descriptive n'a permis de corréler l'expression de Bcl-2 ou de Bcl-xl à la sélection positive des neurones ou, à l'inverse, l'expression de Bcl-xs et leur sélection négative. Les outils nécessaires pour ce type d'étude sont aujourd'hui disponibles et devraient permettre de tester cette hypothèse prochainement.

Dans cette famille de protéines, il existe aussi des protéines dont la fonction est de se comporter comme des antagonistes de Bcl-2. C'est le cas de la protéine Bax. La surproduction de cette protéine dans une lignée de lymphocytes accélère le processus de mort cellulaire induit par la privation 


\section{RÉFÉRENCES}

14. Boise LH, Gonzales-Garcia M, PostemaCE, Ding L, Lindsten T, Turka LA, Mao X, Nunes G, Thompson CB. bcl-x, a $b c l-2$ related gene that functions as a dominant regulator of apoptotic cell death. Cell 1993; 74: $597-608$.

15. Oltvai ZN, Milliman CL, Korsmeyer SJ. $\mathrm{Bcl}-2$ heterodimerizes in vivo with a conserved homolog, Bax, that accelerates programed cell death. Cell 1993; 74:609-19.

16. Borner C, Martinou I, Mattman C, Irmler M, Schaerer E, Martinou JC, Tschopp J. Bcl-2 lacking the membrane attachment site shows increased survival activity in neurons and fibroblasts. $J$ Cell Biol 1994; 126 : 1059 68.

17. Martinou I, Fernandez PA, Missotten $M$, White E, Allet B, Sadoul R, Martinou JC. Viral proteins E1B19K and p35 protect sympathetic neurons from cell death induced by NGF deprivation. I Cell Biol 1995; 128 : 2018.

18. Veis DJ, Sorenson CM, Shutter JR, Korsmeyer SJ. Bcl-2-deficient mice demonstrate fulminant lymphoid apoptosis, polystic kidneys and hypopigmented hair. Cell 1993; $75: 229-40$.

19. Martinou JC, Dubois-Dauphin M, Staple JK, Rodriguez I, Frankowski H, Missoten M, Albertini P, Talabot D, Catsicas S, Pietra C, Huarte J. Overexpression of Bcl-2 in transgenic mice protects neurons from naturally occurring cell death and experi-
mental ischemia. Neuron $1994 ; 13: 1017-30$.

20. Martinou JC, Frankowski H, Missotten M Martinou I, Potier L, Dubois-Dauphin M. Bcl-2 and neuronal selection during development of the nervous system. $J$ Physiol (Pans) 1994; 88: 209-11.

21. Farrow SN, White JHM, Martinou I, Raven $T$, Tao Pun $K$, Grinham $C J$, Martinou JC, Brown R. CEBP-1, a cellular target for E1B19K, is a Bcl-2 homologue (soumis pour publication).

22. Hockenbery DM, Oltvai Z, Yin XM, Milliman $\mathrm{C}$, Korsmeyer S J. Bcl-2 functions in an antioxidant pathway to prevent apoptosis. Cell 1993; 75 : 241-51.

23. Kane DJ, Sarafian TA, Anton R, Hahn H, Gralla EB, Valentine IS, Ord T, Bredesen DE. Bcl-2 inhibition of neural death: generation of reactive oxygen species. Science $1993 ; 262: 1274-7$

24. Kahn A. Bcl-2 inhibe-t-il l'apoptose en s'opposant à l'action des radicaux oxygène. médecine/sciences 1994 ; 10 : 208-9.

25. Lafon-Cazal M, Pletri S, Culcasi M, Bockaert J. NMDA-dependent superoxide production and neurotoxicity. Nature 1993; d'interleukine 3 [15]. Cette protéine est capable de former des homodimères (Bax-Bax) ou des hétérodimères avec $\mathrm{Bcl}-2$ (Bax-Bcl-2). Les domaines de similitude entre $\mathrm{Bcl}-2$ et Bax sont essentiels pour cette dimérisation. Une mutation de Bcl-2, dans l'un de ces domaines, altère la capacité de la protéine de dimériser avec Bax et celle de protéger les cellules. Ces résultats permettent de penser que la fonction protectrice de Bcl-2 pourrait se limiter à la neutralisation de Bax (figure 2).

Parmi les protéines antiapoptotiques connues, il faut aussi mentionner des protéines d'origine virale, certaines apparentées à Bcl-2, d'autres ayant peu ou pas d'analogie avec Bcl-2, comme les protéines E1B19 et $55 \mathrm{~K}$ de l'adénovirus et la protéine p35 du baculovirus. Nous avons montré que E1B19K et p35 pouvaient bloquer la mort cellulaire programmée dans les neurones sympathiques privés de NGF [16]. Les mécanismes d'action de ces protéines ne sont pas encore connus. Toutefois, la fonction de E1B19K pourrait s'apparenter à celle de Bcl2. En effet, E1B19K se lie à une nouvelle protéine de la famille $\mathrm{Bcl}-2$, CEBP-1, dont la fonction, comme celle de Bax, est d'accélérer le processus de mort cellulaire dans des neurones sympathiques après retrait du NGF du milieu de culture [21].

\section{Effecteurs du programme de mort cellulaire}

Une meilleure connaissance du mécanisme d'action des protéines antiapoptotiques que nous venons de décrire devrait permettre d'identifier les mécanismes impliqués dans la mort cellulaire programmée.

\section{- Mort cellulaire et oxydation}

Les observations faites sur les souris chez lesquelles le gène $b c l-2$ a été inactivé permettent d'entrevoir une fonction possible de Bcl-2. En effet ces souris, en plus d'anomalies sévères du développement de la rate, du thymus et des reins, toutes liées à une mort cellulaire excessive, présentent une pigmentation anormale des poils en rapport avec une anomalie de synthèse de la mélanine $\left(m / s n^{\circ} 2\right.$, vol. 10, p. 208) [18]. Cette anomalie a été mise sur le compte d'un déséquilibre du potentiel redox dans les mélanocytes, assignant à $\mathrm{Bcl}-2$ une fonction antioxydante $\left(\mathrm{m} / \mathrm{s} n^{\circ} 2, \mathrm{vol} .10, p\right.$. 208). D'autres études ont confirmé cette fonction en démontrant que Bcl-2 pouvait empêcher, soit la formation de radicaux libres, soit la peroxydation de lipides dans des lignées lymphocytaires ou nerveuses [22-24]. Ces données sont intéressantes, non seulement parce qu'elles révèlent peut-être une des fonctions de $\mathrm{Bcl}-2$, mais aussi parce qu'elles mettent l'accent sur le rôle important que joue l'oxydation dans l'apoptose. La formation de radicaux libres a été mise en évidence dans de nombreux modèles de mort de neurone, en particulier dans des modèles d'excitotoxicité du glutamate [25] $(\mathrm{m} / \mathrm{s}$ $n^{\circ} 6-7$, vol. 10, p. 722). Le maintien du potentiel redox semble essentiel pour la survie cellulaire et il faut souligner qu'une des fonctions vitales des facteurs neurotrophiques est de régler l'activité enzymatique de la glutathion peroxydase, l'enzyme chargée de détoxifier le peroxyde d'hydrogène par l'intermédiaire du glutathion réduit. Nous avons montré que différents antioxydants étaient capables de retarder la mort cellulaire des neurones sympathiques cultivés en absence de NGF (P.A. Fernandez, soumis pour publication). Ces résultats suggèrent que l'un des événements majeurs de la mort cellulaire programmée induite dans les neurones après suppression du NGF est la production de radicaux libres.

\section{- Mort cellulaire programmée et protéases}

Une autre fonction possible pour Bcl-2 est celle d'inhibiteur de protéases et en particulier de protéases à cystéine. La protéine Ced-3, nécessaire au déroulement de la mort cellulaire chez le ver, est similaire à la protéine ICE $\left(\mathrm{m} / \mathrm{s} n^{\circ} 2\right.$, vol. 10, p. 232) [26], une protéase à cystéine d'un nouveau type, responsable de la conversion de la pro-interleukine-1 $\beta$ en interleukine $1 \beta$, et à Nedd-2/ ICH-1 [26, 27], une protéine nouvellement identifiée. Ces trois protéines ont en commun environ $30 \%$ d'acides aminés et, en particulier, possèdent dans leur séquence le 

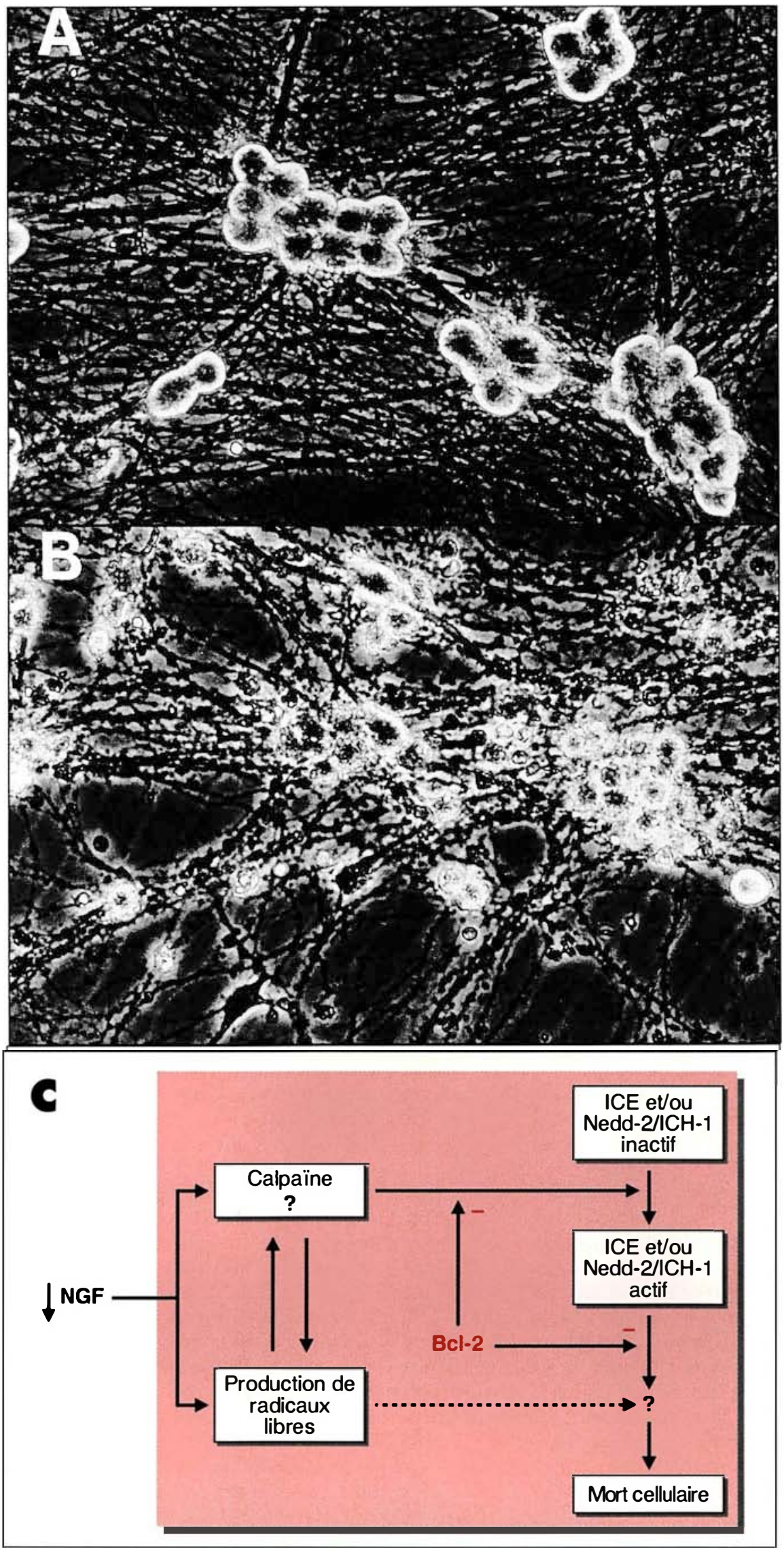

$m / s n^{\circ} 3$, vol. 11 , mars 95

Figure 3. Effecteurs de la mort cellulaire programmée dans des neurones sympathiques privés de NGF. A: Les neurones sympathiques dépendent du NGF pour leur survie. B: Si on les prive de NGF, ils activent un programme de mort cellulaire et dégénèrent. C: La formation de radicaux libres ainsi que l'activation d'une série de protéases à cystéine sont des événements clés de ce programme. Nous pensons que $\mathrm{Bcl}-2$ bloque l'activation ou l'activité des protéases Nedd2/ICH-1 ou ICE. 


\section{RÉFÉRENCES}

26. Yuan J, Shaham S, Ledoux S, Ellis HM, Horvitz HR. The $C$. elegans cell death gene ced-3 encodes a protein similar to mammalian interleukin-1 $\beta$-converting enzyme.Cell $1993 ; 75: 641-52$.

27. Kumar S, Kinoshita M, Noda M, Cope land NG, Jenkins NA. Induction of apoptosis by the mouse nedd 2 gene, which encodes a protein similar to the product of the Cae norhabditis elegans cell death gene ced-3 and the mammalian IL-1 $\beta$-converting enzyme. Genes Dev 1994; 8: 1613-26.

28. Wang L, Miura M, Bergeron L, Zhu $\mathbf{H}$ Yuan J. Ich-I, an Ice/ced-3-related gene, encodes both positive and negative regulators of programmed cell death. Cell 1994: 78 . of progra

29. Gagliardini V, Fernandez PA, Lee RKK Drexler HCA, Rotello RJ, Fishman MC Yuan J. Prevention of vertebrate neurona death by the crmA gene. Science 1994; 263 826-8.

30. Squier MKT, Miller ACK, Malkinson AM Cohen IJ. Calpain activation in apoptosis. Physiol 1994; 159: 229-37.

31. Kahn A, Briand P. L'apoptose, une mort programmée ou une profiferation avortée? médecine/sciences $1993 ; 9$ : 663-5.

32. Golstein P. Mort programmée et terrain cellulaire. médecine/sciences 1991; 7: 681-8.

33. Feddersen RM, Ehlenfeldt R, Yunis WS Clark HB, Orr HT. Disrupted cerebellar cortical development and progressive degeneration of Purkinje cells in SV40 T antigen
transgenic mice. Neuron 1992; 9: 955-66.

34. Hammang JP, Behringer RR, Baetge EE, Palmiter RD, Brinster RL, Messing A. Oncogene expression in retinal horizontal cells of transgenic mice results in a cascade of neurodegeneration. Neuron $1993 ; 10: 1197$ 1209.

35. Jacks T, Fazeli A, Schmitt EM, Bronson RT, Goodell MA, Weinberg RA. Effects of an $\mathrm{Rb}$ mutation in the mouse. Nature 1992 ; 359 : 295-300.

36. Freeman RS, Estus S, Johnson EM. Analysis of cell cycle-related gene expression in postmitotic neurons: selective induction of cyclin D1 during programmed cell death. Neuron 1994; 12:343-55.

37. Dubois-Dauphin M, Frankowski H, Tsujimoto Y, Huarte J, Martinou JC. Neonatal motoneurons overexpressing the bcl-2 proto-oncogene in transgenic mice are protec ted from axotomy-induced cell death. Proc Natl Acad Sci USA 1994; 91 : 3309-13.

38. Pollard HA, Dessi F, Moreau J, Lasbennes F, Ben Ari Y, Charriaut-Marlangue C. Regional variability in DNA fragmentation after global ischemia evidenced by combined histological and gel electrophoresis observations in the rat brain. $J$ Neu- même pentapeptide QACRG*. Ce pentapeptide correspond au site catalytique de ICE et la cystéine qu'il renferme est essentielle à l'activité protéolytique. Il est donc vraisemblable que Ced3 et Nedd2/ICH-1 soient, comme ICE, des protéases à cystéine. ICE est synthétisée sous forme de pré-proenzyme dont le clivage de la partie N-terminale aboutit à la formation d'une proenzyme. Cette dernière peut s'autoactiver par clivage en deux sous-unités de 10 et $20 \mathrm{kDa}$, capables de dimériser pour donner la forme active de l'enzyme. Il apparaît donc qu'une étape essentielle de l'activation de cette enzyme a lieu au niveau post-traductionnel. $\mathrm{La}$ recherche de la protéase responsable de cette activation est d'autant plus importante que l'on pense que Ced-3 et Nedd-2/ICH-1 sont réglées de la même manière qu'ICE. Par ailleurs, l'identification des substrats de ces protéases devrait apporter un élément essentiel à la compréhension des mécanismes de la mort cellulaire programmée.

La surexpression de ICE ou de Nedd-2/ICH-1 induit la mort dans différents types cellulaires [25-27]. L'activité de ces protéases peut être inhibée spécifiquement par une protéine d'origine virale nommée CrmA $[26,28]$. La surexpression de CrmA bloque la mort cellulaire programmée dans des neurones sympathiques, suggérant que l'activation de ICE ou de Nedd-2/ICH-1, voire d'autres membres de cette famille, constitue une étape indispensable dans la mort cellulaire programmée $\left(m / s n^{\circ} 2\right.$, vol. 10, p. 233) [29]. On attend avec beaucoup d'impatience de connaître le phénotype de souris chez lesquelles les gènes ICE et nedd-2/ICH-1 auront été inactivés.

Outre les protéases à cystéine que nous venons de décrire, il faut citer l'implication de protéases à cystéine d'un autre type, les calpaïnes. Il s'agit de protéases dont l'activation est contrôlée par le calcium. L'utilisation d'inhibiteurs de ces protéases a permis de bloquer la mort cellulaire programmée dans des thymocytes [30] ainsi que dans les neurones sympathiques (P.A. Fernandez, soumis pour publication). Ces résultats sug-

* Code des acides aminés: Q: Gln; A: Ala; C: Cys; R: Arg; G: Gly. gèrent que la calpaīne pourrait aussi participer au programme de mort cellulaire. Les résultats présentés dans ce paragraphe sont résumés dans la figure 3.

\section{Mort des neurones et cycle cellulaire}

L'idée que mitose et apoptose partageraient des mécanismes d'action similaires a été énoncée pour différents types cellulaires dans lesquels c-Myc, p53 ou la kinase du cycle cellulaire $\mathrm{p} 34^{\mathrm{cdc} 2}$ ont été impliquées ( $m / s n^{\circ} 9$, vol. $8, p$. 1002). Différentes études faites avec des cellules capables de proliférer permettent d'envisager la mort celullaire comme l'aboutissement d'un échec de la cellule à accomplir une mitose ou comme l'aboutissement d'une mitose commencée dans des conditions inappropriées (absence de facteurs trophiques par exemple) [31, 32]. Qu'en est-il des neurones, cellules post-mitotiques par excellence? Différents auteurs ont montré que la surexpression de l'oncogène SV40 dans des souris transgéniques provoquait une dégénérescence des cellules de Purkinje du cervelet ou encore des cellules horizontales de la rétine $\left(m / s n^{\circ} 2, v o l .9, \quad\right.$ p. 230) [33, 34]. En outre, Jacks et al. (Boston, MA, USA) ont montré que l'inactivation du gène du rétinoblastome, un gène "suppresseur de tumeur", s'accompagnait d'une mort importante des motoneurones et des neurones sensoriels $\left(\mathrm{m} / \mathrm{s} n^{\circ} 10\right.$, vol. $\left.8, p .1116\right)$ [35]. La cause de cette mort a été imputée à l'incapacité de ces cellules de sortir du cycle cellulaire pour se différencier. De ces résultats a émergé l'idée que la mort de neurones serait due à une tentative avortée de réactivation du cycle cellulaire [31]. De nombreuses équipes ont étudié l'expression de marqueurs du cycle cellulaire dans les neurones au cours de la mort cellulaire programmée. Freeman et al. ont montré que le niveau des ARNm de la cycline D1 augmentait environ 10 fois dans les neurones sympathiques privés de NGF [36]. Bien que ces auteurs n'aient pas démontré le rôle de cette cycline dans la mort cellulaire programmée, ce résultat est en accord avec l'hypothèse que des étapes sont communes à la mitose et à l'apoptose. Enfin, on peut 
citer le rôle du proto-oncogène c-Fos dont l'expression prolongée a été associée à la mort des neurones au cours du développement. Malgré toutes ces corrélations, la démonstration que des composants du cycle cellulaire jouent un rôle important dans la mort cellulaire programmée des neurones, voire d'autres types cellulaires, reste à apporter.

\section{Mort cellulaire programmée et maladies neurodégénératives}

Les mécanismes impliqués dans la mort cellulaire responsable des maladies neurodégénératives, en particulier la maladie d'Alzheimer, sont encore mal connus. En raison de la difficulté, voire l'impossibilité de faire des études morphologiques de cellules en train de dégénérer, il est particulièrement difficile de démontrer que la mort cellulaire programmée intervient dans cette maladie. Toutefois, il est important de noter que la mort cellulaire programmée a été décrite dans plusieurs modèles expérimentaux de mort cellulaire en rapport avec des maladies neurodégénératives. Par exemple, la mort cellulaire programmée a été décrite dans la toxicité du glutamate $(\mathrm{m} / \mathrm{s}$ $n^{\circ} 6-7$, vol. 10, p. 722) (modèle de sclérose latérale amyotrophique) ou encore dans la toxicité du MPTP (1-méthyl-4-phényl tétrahydropyridine, $\mathrm{m} / \mathrm{s}$ $n^{\circ} 2$, vol. 1, p. 104 et $n^{\circ} 4$, vol. 3, p. 243) (modèle de maladie de Parkinson). Il faut espérer que la découverte récente de l'apolipoprotéine E comme facteur de risque de la maladie d'Alzheimer permettra le développement rapide de modèles animaux, tant attendus pour la compréhension de la mort de neurones dans cette maladie $\left(\mathrm{m} / \mathrm{s} n^{\circ} 4\right.$, vol. $\left.10, p .470\right)$. Jusqu'à présent, l'essentiel des efforts a porté sur l'étude de la toxicité in vitro du peptide $\beta$-amyloïde qui est un des constituants essentiels des plaques séniles présentes en abondance dans le cerveau des patients atteints de la maladie. Le groupe de Cottman a montré que le peptide $\beta$-amyloïde induisait la mort cellulaire programmée dans des neurones du cortex cérébral.

L'utilisation de souris transgéniques dont les neurones surexpriment $b c l-2$ nous a permis de tester les effets de la protéine $\mathrm{Bcl}-2$ dans différentes si- tuations pathologiques. Nous avons constaté que les neurones de ces souris étaient protégés contre la mort induite par l'axotomie [37], ou encore étaient insensibles à la toxicité du peptide $\beta$-amyloïde. Enfin, dans un modèle expérimental d'ischémie corticale permanente, Bcl-2 était capable de diminuer d'environ $50 \%$ le volume de la région du cortex cérébral ischémique [19]. L'effet protecteur de Bcl-2 était essentiellement marqué dans la région qu'on appelle la pénombre et qui se situe à la périphérie du territoire nécrosé. Ces résultats suggèrent que la mort cellulaire programmée intervient dans le phénomène périlésionnel de "pénombre" comme cela a été démontré dans des modèles d'ischémie transitoire entraînant une mort retardée des neurones de l'hippocampe [38]. Bien que l'ischémie cérébrale ne fasse pas partie des maladies neurodégénératives, on peut ainsi penser qu'elle a en commun avec celles-ci certains mécanismes moléculaires de mort cellulaire.

\section{Conclusions}

La mort cellulaire programmée est un processus de mort cellulaire qui met en jeu un ou des programmes bien codifiés. La liste des différentes protéines qui participent à ce programme est loin d'être close et les mécanismes d'action de ces protéines sont encore largement inconnus. Les progrès rapides réalisés dans ce domaine permettent d'espérer l'identification prochaine de cibles thérapeutiques permettant de développer des molécules antiapoptotiques pour les maladies neurodégénératives. Le succès de ces molécules dépendra de leur capacité d'assurer non seulement la survie des neurones mais aussi le maintien de leur fonction

\section{Remerciements}

Je tiens à remercier $\mathrm{C}$. Hebert pour la réalisation des illustrations de ce manuscrit et les Drs S. Catsicas, J. Knowles, J.J. Mermod et R. Sadoul pour leurs conseils avisés sur la rédaction du manuscrit.

\section{TIRÉS À PART}

\section{Summary}

Programmed cell death in the nervous sytem

Programmed cell death (PCD) or apoptosis is fundamental to the development and maintenance of multicellular organisms. During the development of the nervous sytem, up to 50 percent of many types of neurons normally die during synaptogenesis. This massive death is, at least in part, regulated by epigenetic factors such as neurotrophic factors. The neurons that receive enough trophic factors survive, the others die. These survival signals seem to act by suppressing an intrinsic cell suicide program. Today, very little is known about the molecular and biochemical basis of PCD. Studies in the nematode Caenorhabditis elegans allowed to identify genes that regulate PCD. Two genes, ced-3 and ced-4, are required for neuronal death to occur during development. Another gene, ced-9, acts antagonistically to ced-3 and ced-4 to suppress cell death. The relevance of the studies carried out in $C$. elegans is underscored by the recent identification of mammalian homologues for both the Ced-9 anti-death and Ced-3 death proteins. Ced-9 is homologous to $\mathrm{Bcl}-2$, a suppressor of PCD. Ced-3 was found homologous to the mammalian interleukin $1 \beta$ converting enzyme (ICE), a cysteine protease, and to a recently identified protein called Nedd-2 or ICH-1, both of which can induce PCD in different cell types. Due to their homology with ICE, it is probable that Ced-3 and Nedd-2/ICH-1 are also cysteine proteases. In addition to proteases, free radicals and cell cycle kinases have been also suspected to be mediators of PCD. The molecular mechanisms by which death and anti-death proteins modulate PCD remain unknown. A dysregulation of the balance between these proteins could be the cause of many diseases including neurodegenerative diseases. 